\title{
L'INTERPRÈTE ET LE DEVOIR DE CONCRÉTISER LES OBJECTIFS FONDAMENTAUX DE LA CONSTITUTION
}

\author{
JUAREZ FREITAS ${ }^{*}$
}

\begin{abstract}
RESUMO: No sistema jurídico brasileiro todo juiz é, de certa forma, um juiz constitucional, e deve preservar ao máximo a coexistência pacífica e harmoniosa entre os controles difuso e concentrado de constitucionalidade. A interpretação constitucional é um processo tópico-sistemático, de modo que é imperativo, quando do exame do caso, alcançar uma solução de equilíbrio, evitando soluções unilaterais e buscando ao máximo possível a concretização dos objetivos fundamentais do artigo $3^{\circ}$ da CF.
\end{abstract}

PALAVRAS-CHAVE: Interpretação constitucional. Hermenêutica jurídica. Direitos fundamentais.

ABSTRACT: In the Brazilian legal system every judge is, in a certain fashion, a constitutional judge and the peaceful and harmonious coexistence of the diffuse and concentrated constitutional review systems has to be preserved. Constitutional interpretation is a topical-systematic procedure and therefore it is imperative, during the examination of the case at hand, to reach a balance-solution, avoiding one-sided solutions and trying as much as possible to accomplish the Constitution's article 3 fundamental objectives. KEYWORDS: Constitutional interpretation. Legal Hermeneutic. Fundamental Rights.

RÉSUMÉ: Dans le système juridique brésilien, tout juge est, d'une certaine façon, un juge constitutionnel, et il faut préserver au maximum la coexistence pacifique et harmonieuse entre les contrôles diffus et concentrés de constitutionnalité. L'interprétation constitutionnelle est un processus topique-systématique, de sorte qu'il est impératif, lors de l'examen des cas, d'atteindre une solution d'équilibre, en évitant des solutions unilatérales et en cherchant le plus possible la concrétisation des objectifs fondamentaux de l'article 3ème de la CF. MOTS-CLÉS: Interprétation constitutionnelle. Herméneutique Juridique. Droits fondamentaux.

SUMÁRIO: I. Considerações preliminares; II. Preceitos de exegese constitucional para a concretização dos objetivos constitucionais fundamentais; III. Conclusões.

SUMMARY: I. Preliminary considerations; II. Constitutional exegesis precepts for the accomplishment of the fundamental constitutional objectives; III. Conclusions.

SOMMAIRE: I. Considérations préliminaires; II. Préceptes d'exégèse constitutionnelle pour la concrétisation des objectifs constitutionnels fondamentaux; III. Conclusions.

\footnotetext{
* Professeur à la PUCRS et à la UFRGS, Président de l’Institut Brésilien de Hautes Etudes en Droit Public. Justo agradecer, por suas contribuições à versão francesa, a Janice Sfreddo e a Rita Dostal Zanini, embora a responsabilidade final seja do Autor.
} 


\section{CONSIDÉRATIONS PRÉLIMINAIRES}

L'interprétation de la Constitution, dans le but de lui donner une vie et une réalité nettement axiologique, signifie promouvoir l'incarnation des droits fondamentaux lors de la juste, compétente et efficace promotion du dévéloppement humain. ${ }^{1}$ A partir de clarifications préliminaires, je vais m'appliquer à préciser le choix de certaines prémisses apparaissant comme les matrices du développement de cette exposition. Tout d'abord, il me paraît utile de mettre en évidence le fait que, à mon avis, tout juge, dans le système brésilien, est d'une certaine façon un juge constitutionnel. De ce fait est issu l'engagement de concrétiser la Constitution le plus possible, ainsi que de paraliser une ou autre règle - même si constitutionnelle - si cela se fait nécessaire pour préserver la primauté des principes et droits fondamentaux du système.

En effet, il est urgent d'assumer que chaque magistrat, même au premier dégré, a besoin de continuer doté de l'autorité de juge constitutionnel (sans préjudice du débat tranquille autour des hypothèses très exceptionnelles d'un éventuel contrôle concentré concrètement), sans quoi apparaîtront d'irréparables rétrocessions dans notre façon de contrôler la rationalité inter-subjective, laquelle serait en désaccord avec l'indispensable indépendance de la Magistrature et la consolidation de l'ambiance culturelle de respect volontaire de la suprématie de la Constitution par rapport aux intèrêts particularistes, contraires à l'essentialité propre à la democracie.

Un autre éclaircissement préalable occupe la direction selon laquelle, en termes d'Herméneutique Juridique comme un tout, il faut accueillir la tendance contemporaine (sophistiquée et féconde) d'une synthèse entre deux versants qui, pendant très longtemps, ont été perçus comme irrémédiablement opposés. Je veux parler ici de la conciliation entre la pensée systémique et la méthodologie topique. À chaque avancée, je vois de manière jubilatoire le surgissement de nouveaux arguments destinés à fortifier la perspective philosophique correcte soulignant que l'interprétation juridique est un processus topico-systématique (quelque chose de plus que la "topique liée" de Hesse), dans la lignée de ce que j’ai soutenu dans un ouvrage spécifique ${ }^{2}$. De cette manière, il me semble impérieux de contribuer à une solution d'équilibre, un moyen terme (mesótes aristotélique, qui n'est pas exactement dans le milieu arithmétique), un compromis entre le formalisme et le dénommé empirisme, en s'esquivant des solutions unilatérales et rigides lors de l'examen des cas concrets. De fait, chaque fois qu'un excès d'accentuation polarisante se succède, nombre de maléfices apparaissent. Dans cette optique, il ne faut sous aucun prétexte hypertrophier le formalisme (en dépit d'être l'un des éléments nucléaires de la juridicité) au point de laisser de côté les préoccupations matérielles et empiriques légitimes et rapprochées, d'autant plus que, qu'on le veuille ou non, nous sommes dans une large mesure empiristes, ce qui n'implique pas l'adhésion au scepticisme qui n'utilise que les arguments stratégiques et nie les droits légitimes. ${ }^{3}$

\footnotetext{
${ }^{1}$ Voir Juarez Freitas in Discricionariedade Administrativa e o Direito Fundamental à Boa Administração Pública. São Paulo: Malheiros, 2007, sur le sujet dans le domaine des rapports de gestion.

${ }^{2}$ Voir Juarez Freitas in A Interpretação Sistemática do Direito, 4e éd., São Paulo, Malheiros Editores, 2004.

${ }^{3}$ Voir la critique à un certain type de pragmatisme radical, faite par Ronald Dworkin in Law’s Empire. Harvard: Harvard University Press, 1986.
} 
La seconde observation initiale porte sur le fait que les normes strictes ou règles perdent, chaque fois davantage, de l'espace et du relief au profit des principes fondamentaux (pas nécessairement les généraux), montrant par définition ces derniers comme supérieurs aux premiers, bien que l'on ne doive pas postuler un système uniquement constitué de principes, erreur analogue à celle de prétendre un ordre agissant comme une simple agglutination déconnectée de règles. Didactique, Norberto Bobbio l'a parfaitement signalé: "La crescente importanza che si viene riconoscendo ai principi generalli nella legislazione e nella giurisprudenza deriva dal riconoscimento della loro funzione insieme propellente e delimitante al vertice di un sistema : la quale non esclude peraltro la funzione unificante in campo più strettamente dottrinale”. ${ }^{4}$

La consolidation de la systématicité se fait par la force de l'amalgame uniquement fournie par la nature et par l'action des principes fondants et fondés de l'ordonnancement juridique, et ainsi notre refus de travailler avec la logique du "tout ou rien” dans n'importe quel secteur du Droit semble cohérente ${ }^{5 .}$

La dernière observation préliminaire, que je vais suivre fidèlement et que je préconise dans les études consacrées à l'Herméneutique Juridique en général ${ }^{6}$, repose - en dépit de différences sectorielles - sur la perspective suivante: l'interprétation juridique apparaît comme processus unique, au sein duquel les lacunes sont traitables en tant qu'antinomies spéciales et le principe de la hiérarchisation topique et systématique joue un rôle déterminant, phénomène clairement vérifiable dans le champ constitutionnel, ce qui implique la compréhension ouverte et principiologique des droits fondamentaux ${ }^{7}$, de façon à concrétiser le plus possible tels droits, selons les termes des objectifs constitutionnels fixés dans l'article $3^{\text {ème }}$ de la Constitution Fédérale Brésilienne.

\section{PRÉCEPTES D’EXÉGÈSE CONSTITUTIONNELLE POUR LA CONCRÉTISATION DES OBJECTIFS CONSTITUTIONNELS FONDAMENTAUX}

Ces éclaircissements ayant été réalisés, il est urgent de suggérer une liste non-exhaustive de préceptes d'interprétation, et ce en prenant tout particulièrement en compte la réalité brésilienne et la concrétisation des objectifs prévus dans l'article 3ème de la Constitution, à savoir: construir une société libre, juste et solidaire; garantir le dévéloppement national; éliminer la pauvrété et la marginalisation et réduire les inégalités sociales et régionales et promouvoir le bien de tous, sans préjugés d'origine, de race, de sexe, de coleur, d'âge et tout autre forme de discrimination. Il n’est pas inutile de répéter: l’interprète topique-systématique de la Constitution est celui qui, par-dessus tout, sait élire des directives suprêmes, notamment celles qui composent la table de critères interprétatifs capables de présider n'importe quel type de travail d'application du Droit. De là l'intérêt d'effectuer une proposition de liste

${ }^{4}$ Contributi ad un Dizionario Giuridico, Turin, G. Giappichelle Editore, 1994, p. 279.

${ }^{5}$ Voir, sur ce point, convergence avec Robert Alexy in Teoria dos Direitos Fundamentais. São Paulo: Malheiros, 2008, p. 502.

${ }^{6}$ En particulier dans A Interpretação Sistemática do Direito, ob. cit.

${ }^{7}$ Dans ce point spécifique, il y a une autre convergence avec Robert Alexy in ob. cit., p. 543. 
de préceptes ou de critères qui sont ou devraient être prioritaires dans le domaine de l'exégèse constitutionnelle, tout en considérant, emphatiquement, les progrès dans le champ de la doctrine philosophique d'Herméneutique Juridique.

Plus que jamais, force est de reconnaître que dans la fixation ou le dévoilement de telles directives aucun avantage ne résulte de la conception anachronique de Constitution comme ensemble normatif par rapport auquel l'exégète inférerait des solutions de manière exclusivement déductive. Sur le plan factuel, il n’y a aucun sens à imaginer que l'interprète constitutionnel - et dans une certaine mesure, tout juge l'est - réussisse en ne travaillant qu'avec des modèles subsumés logico-formellement. En termes contemporains, la meilleure voie concerne le choix convenable des prémisses adéquates et nécessaires tout au long du chemin de compréhensiondécision; processus qui, en essence et selon Gadamer, ne doit jamais expérimenter une coupure rigide, sous peine d'une approche infidèle du monde réel. L'on se souvient tout particulièrement, à ce propos, de la leçon judicieuse et inattaquable de Hans-Georg Gadamer: "La tâche de l'interprétation consiste en la concrétisation de la loi dans chaque cas, c'est-à-dire dans son application. La complémentation productive du Droit, qui a lieu avec cela, est évidemment réservée au juge, mais celui-ci se trouve, à son tour, sujet à la loi, exactement comme n'importe quel autre membre de la communauté juridique. Dans l'idée d'un ordre juridique, il est supposé que la sentence du juge ne surgit pas d'arbitraires imprévisibles mais d'une pondération juste de l'ensemble" ("Die Aufgabe des Auslegens ist die Konkretisierung des Gsetzes im jeweiligen Fall, also die Aufabe der Applikation. Die Leistung produktive Rechtergänzung, die damit geschieht, ist gewiss dem Richter vorbehalten, der aber genau so unter dem Gesetz steht wie jedes andere Glied der Rechtsgemeinschaft. In der Idee einer rechtlichen Ordnung liegt, dass das Urteil des Richters nicht einer unvorhersehbaren Willkür entspringt, sondern der gerechten Erwängung des Ganzen”). ${ }^{8}$

Dans la pratique nos syllogismes juridiques sont les dialectiques. Dans la lignée de ce qui est dévoilé, parmi d'autres, par Claus-Wilhem Canaris ${ }^{9}$, ce qui est important, dans un contexte de décision juridique, résulte de la qualité de l'élection des prémisses. En d'autres termes, le modèle vétuste et insatisfaisant des syllogismes formels est presque entièrement dépassé, du moins au niveau théorique. Cependant, des efforts en vue de le pratiquer, tenaces et pathologiques, persistent et rien n'empêche sa totale impraticabilité, ce qui est, d’ailleurs, sa plus grande réfutation. Il faut donc dépasser, finalement, la vision restreinte et asphyxiante de l'exégèse juridique, en établissant des préceptes matériels supérieurs servant de boussoles, de paramètres ou de références pour notre façon de donner vie à la Constitution et au Droit comme un tout. ${ }^{10}$

Dans la vision topique-systématique ${ }^{11}$, orientée par la concrétisation maximale

\footnotetext{
${ }^{8}$ in Wahreit und Methode. Tübingen: J.C.B. Mohr, 1965, p. 312.

${ }^{9}$ in Systemdenken und Systembegriff in der Jurisprudenz, Berlin, Duncker und Humblot, 1983.

${ }^{10}$ Dans cette perspective, voir Alexandre Pasqualini, Hermenêutica e Sistema Jurídico, Porto Alegre, Livraria do Advogado, 1999.

11 Dans la lignée que la pensée problématique contribue «à une plus ample ouverture du système constitutionnel », voir Emerson Garcia in Conflito entre Normas Constitucionais. Esboço de uma Teoria Geral. Rio: Lumen Juris, 2008, p. 93.
} 
possible des objectifs fondamentaux Constitutionnels, je propose le premier précepte d'interprétation constitutionnelle, celui qui prône: en hiérarchisant prudemment les principes et les normes (au sens de règles) constitutionnelles, nous devons faire en sorte que les principes occupent la place la plus importante pour la concrétisation harmonique des objectifs fondamentaux, si nécessaire paralysant l'application des règlés, même si constitutionnelles. Par exemple, l'on peut paralyser, avec la juste motivation, l'incidence de l'article 100 de la CF pour la préservation du principe fondamental de la dignité et en défense de la vie. ${ }^{12}$ Les objectifs constitutionnels se placent à la base et au sommet du système, c'est-à-dire en les considérant, dans la pratique, comme fondement et sommet de celui-ci. En effet, dans la mesure où il n'y a pas d'hypothèse écartant la hiérarchisation (toujours interpréter hiérarchiser et ordener ${ }^{13}$ ), il importe de percevoir que l'impossibilité d'écarter la hiérarchisation transforme le critère hiérarchique matérielle en directive opérationnelle supérieure aux autres critères (chronologique et de la spécialité); d'où la nécessité d'assumer également les conséquences de cette toute-puissance hiérarchisante, notamment lorsque nous avons affaire au phénomène de la collision de principes et, du reste, aux dites antinomies de second degré.

Hiérarchiser est donc la note suprême de l'interprétation constitutionnelle. Lorsque l'on hiérarchise les principes et les règles constitutionnels, le rôle réalisateur de l'interprète transparaît plus clairement, à savoir d'être celui qui donne vie à l'ordonnancement, en particulier aux objectifs fondamentaux de la Constitution, sans proprement le convertir en législateur invahissant, même si, dans les cas excepcionnels, le précédant vincule avec une force qui équivaut à celle de la loi, et, dans d'autres cas, le mandat d'injonction remplace provisoirement l'activité du législateur. Dans ce cas, il ajoute une norme au système jusqu'à ce que le législateur accomplisse son rôle institutionnel. ${ }^{14}$ Ainsi la polémique est-elle dépassée, sans sens dialectique, entre objectivisme et subjectivisme. Il est préférable d'affirmer que l'interprète constitutionnel en général (et, de manière plus importante, le magistrat), d'une certaine façon, positive finalement le Droit. Nul doute que c'est le juge (et non le législateur) qui achève le processus de positivation juridique de la procédure.

Par conséquent, la jurisprudence, même dans le système roman, doit être désormais distinguée au minimum comme la source matérielle la plus significative, tel que l'entend d'ailleurs et très justement, notamment, García de Enterria ${ }^{15}$, qui énonce des remarques basilaires: a) "Concretamente, el Derecho no pude ya considerarse como la voluntad del Estado, puis implicaría dejar fuera todo el sistema de fuentes formalmente subsidiarias llamadas a aplicarse justamente en la hipotésis de una ausencia o de una deficiencia de esa voluntad”16, car, de fait, si le Droit n’était qu'étatique, cela équivaudrait à la fin de son ouverture génétique ; b) dans la lignée

${ }^{12}$ Voir, par exemple, le jugement par le STJ du Agravo Regimental no Recurso Especial 2006/0211753-6.

${ }^{13}$ Voir, sur le principe systématisateur (« Ordnungsidee »), Eberhard Schmidt-Assmann in Das Allgemeine Verwaltungrescht als Ordnungs Idee. Heidelberg: Springer Verlag, 1998.

${ }^{14}$ Voir, par exemple, jugement par le STF sur les Mandados de Injunção 670, 708 e 712.

${ }^{15}$ Reflexiones sobre la Ley y los Principios Generales del Derecho, Madrid, Civitas, 1984.

${ }^{16}$ Idem, p. 19. 
de ce que nous soutenons, le choix du critère interprétatif est un prius logique pour pouvoir aborder la compréhension d'un texte ${ }^{17}$; c) les principes généraux sont les seuls instruments susceptibles de donner du sens aux institutions, dominant entièrement les opérations mentales d'interprétation ${ }^{18}$; d) les principes sont désormais vus comme l'expression d'une justice matérielle, néanmoins spécifiée en fonction des problèmes juridiques concrets, ainsi admise une conception substantialiste du Droit ${ }^{19}$; e) d'autre part, "la Ciencia Juridica no tiene outra misión que la de desvelar y descubrir, a través de conexiones de sentido cada vez más profundas y ricas, mediante la construcción de instituciones y la integración respectiva de todas ellas en un conjunto, los principios generales sobre los que se articula y debe, por conseguiente, expresarse el orden jurídico". ${ }^{20}$ Il convient d'ajouter la quête de l'efficacité maximale possible $^{21}$ dans la défense de la systematicité, sous peine de prise de décision imodéré et sans le fondemente raisonable, détérminé par l’article 93 de la CF.

Or, si la tâche suprême consiste à hiérarchiser des principes, des règles et des valeurs, pour la concrétisation des objectifs fondamentaux de l'article 3ème de la Constitution, il est manifeste que la mutation constitutionnelle, se produisant à travers la prépondérance changeante de telle ou telle solution pratique apparaît indispensable à la propre santé et cohérence du système dans son organicité vitale et complexe. Par conséquent, l'interprète passif d'autrefois n'est plus admis, même s'il y a encore, peut-être plus raffinés, des nostalgiques de l'École de l'Exégèse. Une telle orientation mécaniciste, ainsi que nous l'avons déjà souligné, est en désaccord total avec la vie réelle. Évidemment qu’il ne s’agit pas de comprendre la hiérarchisation en termes d'application d'un Droit libre. Sans aucun doute, des limites formelles et substantielles existent pour donner une vie socialement adéquate et dogmatiquement consistente à l'ordre juridique, et l'une d'entre elles advient du devoir de réaliser, très précautionneusement, le contrôle de l'exercice du pouvoir discrétionnaire dans la mise-en-pratique et élection des politiques publiques.

Tout en soulignant une telle dimension hiérarchisante, j'adopte consciemment la position "constitutionnaliste" en lieu et place de celle de "légaliste stricte"; autrement dit, pondérer apparaît de toute évidence plus significatif pour l'interprète constitutionnel que subsumer. ${ }^{22}$ Il n'est donc pas surprenant, par exemple, que dans l'action directe d'inconstitutionnalité parfois la liminaire puisse avoir des effets ex tunc et que la déclaration définitive de l'inconstitutionnalité n'opère pas toujours des effets ex tunc (il suffit de songer à la portée de l'article 27 de la Loi brésilienne 9.868, de 1999). Il est évident que modération et parcimonie sont attendues dans l'exégèse de ces dispositifs; mais il n’y a pas de doute que s’ils sont interprétés dans les proportions

\footnotetext{
${ }^{17}$ Idem, p. 23.

${ }^{18}$ Idem, p. 24.

${ }^{19}$ Idem, pp. 30-31.

${ }^{20}$ Idem, p. 24

${ }^{21}$ Voir Paulo Caliendo dans la défense de la «maximale efficacité possible » in Direito Tributário e Análise Econômica do Direito. Uma Visão Crítica. Rio: Campus, 2009, p. 193.

${ }^{22}$ Voir Eros Roberto Grau in A Ordem Econômica na Constituição de 1988, 5e éd., São Paulo, Malheiros Editores, 2000.
} 
dues, ils évitent ou permettent d'éviter le mal majeur de la non-déclaration d'inconstitutionnalité provoqué par la crainte des effets nocifs qu'une éventuelle déclaration non restreinte, inconsidérée et précipitée pourrait provoquer en termes de confiance légitime et sécurité des rapports juridiques, voire de justice matérielle et sociale. Le “donner vie" demande le dépassement de paradoxes apparents. Il n'est pas surprenant que, pour préserver le système, l'on accepte, excepcionnellement, la convalidation juridique de l'acte inconstitutionnel. Il est important de souligner qu'il ne s'agit pas d'admettre l'exception tout court, mais surtout d'affirmer que la défense elle-même du systéme détermine que l'on considére plus attachant le devoir de convalider plutôt que celui de déclarer la nullité. ${ }^{23}$ D’où l'importance de la pensée topique-systématique, qui demande une motivation rigoureuse et consistente, notamment dans les situations de cette nature-là.

En cela nous observons, comme dans tout d'ailleurs, le besoin du juste équilibre préconisé entre formalisme et exigences de la vie réelle. Toutefois, je remarque au passage qu'en prenant justement en compte un tel équilibre dialétique, il ne paraît pas convenable pour l'instant de procéder à l'importation de la figure de "l'appel au législateur” (Appellenstscheidung), car cela mènerait à des excès susceptibles de produire, au Brésil, l'aggravation de l'insécurité juridique et du tableau d'imprévisibilité frénétique.

Afin de conclure cette brève appréciation du premier précepte proposé, force est de retenir que bien interpréter tout dispositif face à la Constitution revient à le mettre, plastiquement, en connexion avec la totalité vivante du système, tout en réalisant, de manière topico-systématique, la hiérarchisation réfléchie des principes, des règles et des valeurs, et ce en vue d'obtenir la plus grande justice possible, substantiellement indispensable à la vie des objectifs fondamentaux de la Constitution. Il convient ici de reprendre la leçon de Norberto Bobbio ${ }^{24}$ : "non sarà inutile richiamare l'attenzione sul fatto che l'interpretazione sistematica è tra i modi di argomentazione giuridica quella cui si attribuirse maggiore forza probativa”. Mais elle est bien plus que cela: c'est elle qui résoud les antinomies apparamment insolubles entre les critères pour résoudre les antinomies et elle atteste le sérieux des objectifs les plus élévés du système.

Le second précepte repose sur le fait que l'interprète constitutionnel doit être le gardien d'une perspective proportionnelle de la Constitution, cette proportionnalité n'étant pas comprise comme une adéquation moyen-fin. Ladite proportionnalité interdit, en même temps, l'arbitraire par excès et par omission. La proportionnalité signifie notamment que nous sommes obligés de sacrifier le minimum afin de préserver le maximum de droits. Cette formule semble éclaircir suffisamment le précepte, le considérant aussi comme quelque chose de plus que la simple interdiction d'excès. Un droit constitutionnel ne doit, en aucune circonstance, supprimer totalement un autre droit et il est inacceptable l'omission qui engendre de préjudices juridiquement injustes, ce qui implique une révision de la théorie sur la responsabilité civile de

\footnotetext{
${ }^{23}$ Voir le jugement par le STJ, du RMS 25.652-PB.

${ }^{24}$ Contributti ad un Dizionario Giuridico, p. 290.
} 
l'Etat par omission. ${ }^{25}$ Je rappelle en outre que Badura ${ }^{26}$, tout en reconnaissant le versant administratif du principe de la proportionnalité (pas un simple devoir ni un postulat formel), originairement lié à l'exercice du pouvoir de police administrative, s'est rendu compte de sa transformation en limite général à l'intervention du Pouvoir Exécutif. On avance: l'omission de prestations positives de la part de l'Etat est interdite et l'interprète qui veuille accomplir les objectifs constitutionnels doit en avoir pleine conscience. La Constitution, dans ses objectifs fondamentaux, établit des liens. La non-obéissance signifie la chute du principe de proportionalité en tant qu'interdiction de la négligence ou de l'insuffisance. ${ }^{27}$ Il s'agit d'un acte non-juridique, puisque le principe de la proportionnalité est un droit positif. ${ }^{28}$

Il va de soi qu'on ne peut pas laisser de faire attention à la remarque de Heinrich Scholler à ce propos: "Une survalorisation de la proportionnalité, de son côté, trouve des limites dans le principe de l'isonomie, de sorte que tous les deux (principe de la proportionnalité et de l'égalité de traitement) doivent être appliqués de forme simultanée et harmonieuse. La jurisprudence a développé le contenu du principe de la proportionnalité en trois niveaux : la loi, pour correspondre au principe de la réserve de la loi proportionnelle, devra être à la fois adéquate (geeignet), nécessaire (notwendig) et raisonnable (angemessen)"29.

Un tel principe de proportionnalité, lié aux actes juridiques et administratifs (pas seulement les discrétionnaires) et aussi aux lois (certains affirment avec raison l'existence du renomé principe de la réserve de la loi proportionnelle), naît donc pour restreindre toutes sortes d'abus ou d'arbitraire (par excès et par omission). De cette façon, il n'est pas possible, en l'invoquant, d'empêcher des abus ou des excès en brandissant plus d'abus ou d'excès. Indubitablement, rien ne sert de combattre des disproportionnalités par d'autres encore plus grandes. En effet, le principe de la proportionnalité signifie, en définitive et essentiellement, l'élément suivant: nous devons faire concorder les valeurs juridiques et, lorsque l'une de ces valeurs doit dominer l'autre, il faut sauvegarder au maximum celle qui a été relativisée. Le défi de réaliser une bonne interprétation systématique de la Loi d’Improbité est un exemple de notre préoccupation concernant la manipulation adéquate du principe de la proportionnalité. On ne peut pas, par exemple, considérer toute irregularité comme immoralité, sous peine de se convertir à la juste défense du principe constitutionnel de la moralité sous un moralisme non-justifié. ${ }^{30}$

${ }^{25}$ Sur le rapport entre proportionnalité et responsabilité, voir Discricionariedade Administrativa $e$ o Direito Fundamental à Boa Administração Pública, ob. cit., pp 62-77.

${ }^{26}$ Staatsrechts, Munich, Verlag, Franz Vahlen, 1985, p. 125 et suivantes.

${ }^{27}$ Sur le sujet de « l'interdiction d'insuffisance », voir Ingo Sarlet in « Constituição e proporcionalidade : o direito penal e os direitos fundamentais entre a proibição de excesso e de insuficiência». Revista da Ajuris $n^{\circ}$ 98, p. 132. Voir aussi, Lênio Luiz Streck in «A dupla face do princípio da proporcionalidade ». Revista da Ajuris n. 97, p. 180. Voir, pour illustrer dans le Droit Comparé, Claus-Wilhelm Canaris in Direitos Fundamentais e Direito Privado. Coimbra-Almedina, 2003, pp 132-134.

${ }^{28}$ Voir Paulo Bonavides dans un Chapitre spécifique du Curso de Direito Constitucional. São Paulo: Malheiros Editores, 2007.

${ }^{29}$ in "O princípio da proporcionalidade no direito constitucional e administrativo da Alemanha”. Revista Interesse Público 2/97.

${ }^{30}$ Voir, comme exemple d'exegése proportionnelle, le jugement par le STJ, du Resp 511.095-RS. 
En outre, le principe de la proportionnalité présupose une tension dialétique du système, et non une simple simetrie morfologique. La Constitution de 1988, au-dèlà des idéologies unilatérales, possède des objectifs fondamentaus qui demandent plein respect des tensions sociales et politiques, c'est-à-dire la tension interne de la téléologie constitutionnelle ne constitue pas un défaut, plutôt une vertu, celle d'incorporer des contradictions défiant en permanence des solutions proportionnelles. Par conséquent, la Constitution qui loge des objectifs à prémière vue contradictoires mérite des éloges. Il me semble en effet qu'une Constitution démocratique doit forcément présenter une tension interne innée, sous peine de ne pas traduire, de manière légitime et en permanente légitimation, les facettes de la société par essence pluraliste. D’où advient le besoin d'un travail interprétatif permanent de mise en compatibilité et de donner une vie organisée et proportionnelle (sans excès ni négligence) aux prescriptions fragmentaires, en particulier aux objectifs faisant partie de l'article $3^{\text {ème }}$ de la CF.

Prenons cependant le défi posé par l'article 62 de notre Constitution. La Cour Suprême, en déterminant le contenu des conceptes « relevant » et « urgent » peut interdire les abus du Pouvoir Executif, sans avoir à demander une réforme de la Constitution. (En même temps, avec le surnomé mandat d’injonction, elle peut éviter l'omission du Parlement). Dans les deux cas, la disproportionnalité est non-juridique et ne doit pas être traitée avec passivité, en ce moment de l'Etat démocratique, sous peine d'omission inconstitutionnelle du contrôle judiciaire. Pourtant, dans l'exemple donné, la disproportionnalité dans l'utilisation des mesures provisoires est telle que la Cour devrait faire valoir, de manière plus revendicative, la large possibilité judiciaire d'investigation. En effet, si l'on estime que la mesure extrapole toute limite de proportionnalité, il paraît logique de décider d'emblée de l'écarter, parce qu'arbitraire. Ajoutons que ce serait extrêmement bénéfique si cette posture topique devenait une nouvelle tradition, indépendamment de l'avènement d'une discipline constitutionnelle restrictive de l'édition de mesures provisoires (outre celle déjá apportée par l'Emenda Constitucional 32). C'est dire que, dans des situations analogues, nous devons avancer vers la reconnaissance pleine de la plus grande possibilité d'investigation des lois et des actes normatifs, justement pour une préservation meilleure et effective, notamment du principe de la proportionnalité.

Cela étant dit, c’est le moment de présenter le troisième précepte, à savoir, la directrice interprétative selon laquelle l'interprète constitutionnel doit considérer de manière plus large l'incontournable pouvoir-devoir d'accorder la tutelle, de façon à faciliter, au maximum, l'accès légitime du juridictionné. En d'autres termes, il s'agit de dégager tous les effets de l'adoption, chez nous, du système de juridiction unique, car ce système est une clause irrévocable, d'où suit l'intangibilité de ce qui apparaît dans l'article XXXV de la Constitution Fédérale. Un tel “monopole” de l'exercice de la juridiction ne comporte aucun type de flexibilité, et demande beaucoup d'attention, par exemple, pour l'examen de «repercussion générale ». En cas de doute, il faut faciliter l'accès au STF. ${ }^{31}$ S'il est menacé ou violé, on se trouve face à un non-respect

${ }^{31}$ Voir CF, dans l'article 102, § $3^{\text {o: }}$ dans le cas du « recours extraordinaire », celui qui le fait doit démontrer la « repercussion générale » des questions constitutionnelles discutées dans le cas, afin de permettre que la 
patent de l'item IV de l'article 60, § 4 de la Constitution. En matière de contrôle de la constitutionnalité, c'est le Pouvoir Judiciaire qui doit avoir le dernier mot, qui doit être en faveur des objectifs fondamentaux, jamais une fuite de ce devoir. D'autre part, il incombe seulement au juge, alors qu'il exerce la tutelle juridictionnelle typique, de dire en dernier si l'acte interna corporis l'est de fait, tout en rappelant qu'il n'existe pas théoriquement et à priori un acte exclusivement politique. En conséquence, il n'y a pas, à la rigueur, d'acte qui ne puisse être investigué dans notre système. Il sera toujours possible, au moins, le contrôle du «démérite» des actes administratifs. ${ }^{32}$ Ce qui signifie un approfondissement de la sindicabilité des juridictions.

C'est pourquoi, si nous pouvons être des adeptes conscients de la flexibilité de la pensée systématique, nous devons aussi comprendre que notre système a besoin d'avoir des parties immobiles (on se souvient, avec un léger changement d'acception, de l'expression consacrée de Wilburg) et que le caractère un de la juridiction doit être préservé comme partie fondatrice do système brésilien, dont la tutelle des droits subjectis doit être une puissante alliée de la concrétisation des objectifs fondamentaux de la Constitution, et jamais s'en abstenir. S'il n'en est pas ainsi, l'État Constitutionnel risque de périr, affectée jusqu'au tréfonds. Au sens propre, seul le Pouvoir Judiciaire exerce juridiction, aussi bien dans des procédures subjectives que dans les procédures objectives. Dans ce registre, bien que la Loi d'Arbitrage parle de juge de fait et de droit, seuls les juges exercent la fonction juridictionnelle typique. De cette façon, le juge étant le détenteur unique de la juridiction, il doit agir en tant que guardian par excellence de l'indéniable droit d'accès à la tutelle juridictionnelle: en cas de doute, il doit accorder la préférence à l'exégèse qui élargit l'accès au Pouvoir Judiciaire, aussi congestionné qu'il soit, sans exclure les démarches intelligentes pour le désencombrer, surtout en restreignant les manœuvres de ressources pour ajourner illégitimes et en établissant que le STF doit viser les attributions concernant la condition de Tribunal Constitutionnel, sans le distraire avec des tâches diverses, d'autant que ses attributions sont suffisamment névralgiques pour justifier l'existence de cette Cour ${ }^{33}$.

Le quatrième précepte suggéré est celui qui indique que l’interprète constitutionnel doit maintenir un engagement responsable envers l'effectivité du discours normatif de la Constitution, notamment des objectifs fondamentaux, sans oublier le devoir de rationnalité fiscale. Sous l'égide d'un tel précepte éminemment intégrateur (selon l'acception de Smend), il en résulte que si nous avons un doute, à savoir si nous nous trouvons devant une norme à efficacité pleine, contenue ou limitée, l'exégèse qui mène à la concrétisation adressée à la plénitude est toujours préférable, considérant l'impérativité comme étant toujours présente, même si dans un dégré variable. Comme l’a pertinemment observé Jorge Miranda, “à une norme fondamentale doit être

Cour examine l'admission du recours, ne pouvant le refuser que par la manifestation de deux tiers de ses membres (voir Emenda Constitucional 45). Voir aussi, la Loi 11.418/2006.

${ }^{32}$ Voir Juarez Freitas in Discricionariedade Administrativa e o Direito Fundamental à Boa Administração Pública, ob. cit., en particulier le Chapitre 2.

${ }^{33}$ Voir à ce sujet Clèmerson Clève, A Fiscalização Abstrata da Constitucionalidade no Direito Brasileiro, 2e éd., São Paulo, 2000, p. 409. 
attribuée le sens qui lui donne le plus d'efficacité; à chaque norme constitutionnelle il faut attribuer, liée à toutes les autres normes, le maximum de capacités de réglementation". ${ }^{34}$

En outre, il faut éviter, parmi diverses alternatives, celles qui empêchent toute efficacité immédiate des objectifs fondamentaux. Faute de quoi nous admettrions le contresens de norme sans aucune efficacité. Il est des cas - comme celui du droit de grève des fonctionnaires - où l'on constate un besoin indéniable d'une loi réglementariste. Néanmoins, en cas d'absence de cette dernière, l'interprète devra avoir à l'esprit l'impératif de l'optimisation et, dans le cas du Pouvoir Judiciaire, il pourra combler le vide législatif par le mandat d'injonction, comme déjà vu. Il n’appliquera pas de sanctions aux grévistes de la même manière qu'il le ferait si le droit n'apparaissait pas dans le texte de la Constitution. Donc, l'interprète constitutionnel reconnaît son engagement premier envers l'efficacité ${ }^{35}$, y compris lorsqu'il s'occupe de dispositifs non-autoapplicables. Dans notre système, il est licite d'affirmer qu'il y aura toujours un certain effet venant des préceptes constitutionnels. Il est opportun de renforcer ce qui vient d'être dit par les paroles de Konrad Hesse : "Puisque la constitution prétend être actualisée et étant donné que les possibilités et les conditionnements historiques de cette mise à jour se modifient, il se fera nécessaire, dans la résolution des problèmes, de donner préférence aux points de vue qui, selon les circonstances de chaque cas, aident les normes constitutionnelles à atteindre l'efficacité maximale” (“Da die Verfassung aktualisiert werden will, die geschichtlichen Möglichkeiten und Bedingungen dieser Aktualisierung sich aber wandeln, ist bei der Lösung verfassungrechtlicher Probleme denjenigen Gesichtspunkten der Vorzug geben, die unter der jeweligen Voraussetzungen den Normen der Verfassung zu optimaler Wirkunskraf verhelfen”. ${ }^{36}$

De même, les principes programmatiques et les objectifs fondamentaux de l'article 3ème de la CF ne doivent pas être lus comme s'ils étaient des appels d'une rhétorique vide, frivole, banale ou superflue, mais plutôt comme s'ils portaient une vie d'efficacité, et ce malgré leur fonction projective. Il n’y a rien dans la Constitution qui ne doive répercuter sur la totalité du système juridique et par conséquent sur la vie réelle. Prenons le cas paradigmatique du principe de l'isonomie, sur lequel Paulo Bonavides a tissé des commentaires lucides dans les termes lumineux suivants: “En réalité ce que l'on prétend et ce que l’on doit poser ici, concernant l'égalité des faits, est le problème capital de l'efficacité et de la juridicité du principe; c'est la reconnaissance qu'il est là, admis dans des clauses spéciales d'isonomie dans une Constitution, pour obliger le législateur quel qu'il soit par exemple dans l'échelle fédérative d'un système donné. Notre réponse à ce sujet, qui est intimement liée à l'interprétation de l'article 135 de la Constitution Fédérale est que l'isonomie fonde un postulat d'égalité des faits. Plus qu'une valeur, il est un principe de base de l'ordre constitutionnel, de la Constitution ouverte. (...). L’égalité factuelle est le concept clé

\footnotetext{
34 "Direitos fundamentais e interpretação constitucional", RTRF-4a Região 20/2 an 9.

${ }^{35}$ Dans le cas du Droit Administratif, le principe d'efficacité est consacré dans le texte de la Constitution, dans l'article 74.

${ }^{36}$ Grundzüng des Verfassungrechts der Bundesrepublik Deutschland, Heidelberg, C. F. Müller Juristischer Verlag, 1978, p. 30.
} 
dans l'interprétation de l'équivalence de droits. Forme de l'isonomie matérielle, elle se qualifie juridiquement comme une règle qui oblige l'État, si nécessaire, à des prestations positives (...). Depuis longtemps les droits fondamentaux à teneur égalitaire n’occupent plus dans les Constitutions le lieu abstrait des déclarations programmatiques. Ils ne sont plus simplement un document de bonnes intentions du Constituant, de son idéalisme, de sa générosité. Tout ce qui se trouve dans la Constitution est juridique (...)”. ${ }^{37}$ Plus: l'efficacité du principe d'égalité pressupose la reduction des inégalités illégitimes et pas seulement l'empêchement de discriminations négatives (CF, article 3ème, qui exige la promotion du "bien de tous”). En d'autres mots, il exige l'accomplissement du devoir de réaliser différentiations positives et réductrices des inégalités inconstitucionnelles (CF, article 3, III) condition essentielle pour la promotion efficace du dévéloppement humain. ${ }^{38}$

Toujours ayant un rapport intime avec les autres, le cinquième précepte de l'interprétation constitutionnelle est celui qui souligne que l'interprète doit chercher un fondement rationnel objectif et vital face à ses décisions en dialogue avec le système, sans jamais adopter de solutions “contra legem” et sans parier sur une seule réponse correcte. ${ }^{39}$ Autrement dit, il doit avoir le désir de lutter, avec impartialité, contre tout subjectivisme réducteur de la juridicité du système constitutionnel qui porte un grave préjudice à l’État de Droit.

Or, l'une des plus hautes fonctions de l'ordonnancement réside justement dans le combat à la décision sans motivation consistente, c'est-à-dire sans fondaments de fait et de droit en harmonie avec les objectifs fondamentaux de la Constitution. On ne peut jamais éradiquer une dose de subjectivité salutaire puisque la liberté, heureusement, est un trait inextirpable de l'acte humain de juger, étant donné que nous n’opérons pas avec des syllogismes logico-formels. Toutefois, on doit écarter toute prise de décision irrationnelle mue sous l'influence délétère des passions associées à des motivations inférieures ou non passibles d'universalité, si attrayantes que soient les pragmatiques tentations. Ce n'est qu'ainsi que nous serons capables de révéler, et en même temps d'édifier, un système constitutionnel acceptable face à l' "auditorium universel" dont nous parlait Perelman, constamment soucieux de la responsabilité éthique des décideurs judiciaires. ${ }^{40}$ Principalement en matière de contrôle de la constitutionnalité, une telle considération, qui vise à harmoniser le système juridique et les exigences de la rationalité communicative ${ }^{41}$, se révèle inajournable et il importe de souligner que celui qui juge doit, par-dessus tout, encourager de manière permanente, l'adhésion bien pensée aux objectifs fondamentaux, en n'agissant jamais sous l'emprise de simples impressions, de liens cachés ou, comme déjá dit, d'aspirations non passibles d'universalité.

\footnotetext{
${ }^{37}$ A Constituição Aberta, 2e éd., São Paulo, Malheiros Editores, 1996, p. 109.

${ }^{38}$ A propos du «dévéloppement humain », voir le concepte adopté par le Programme des Nations Unies pour le Dévéloppement.

${ }^{39}$ Pour ce qui est de ce sujet spécifique, en accord avec Robert Alexy in ob.cit, p. 543.

${ }^{40}$ in Ética e Direito. São Paulo: Martins Fontes, 1996.

${ }^{41}$ Voir Jurgen Habermas in Theorie des Kommunikativen Handelns, vs I et II, Frankfurt am Maim: Suhrkamp, 1985.
} 
Dans la continuité de l'exposition de cette série non-taxative de vecteurs donnés d'interprétation constitutionnelle, il convient de citer le précepte de la préservation simultanée des caractéristiques fonctionnellement vitales de tout système démocratique digne de ce nom, à savoir l'ouverture et l'unité. L'interprète constitutionnel ne doit pas méconnaître l'ouverture du système (dans les deux acceptions de Canaris ${ }^{42}$ c'est-à-dire la cognitive et celle du système objectif), car c'est à travers elle (ouverture) dans sa double dimension que le grand moteur préférentiel des mutations constitutionnelles pourra advenir, comme cela se passe déjà dans des pays tels que l'Allemagne et les États-Unis, pour citer des modèles distincts de contrôle de constitutionnalité.

Afin de préserver l'ouverture et l'unité, le juge constitutionnel doit d'abord effectuer, hardiment si nécessaire, les mutations requises en vue de profiter correctement de la flexibilité de la Constitution. A titre d'image, la Constitution est le cœur juridique du pays. Ainsi, moins il y a d'interventions invasives (émanant de l'exercice du pouvoir d'amendement), mieux cela sera pour ce cœur institutionnel. On ne peut pas remettre en question le fait que chaque réforme constitutionnelle fonctionne comme un processus invasif, raison suffisante pour affirmer qu'il est extrêmement préférable, du moins à moyen terme, que l'interprète agisse comme étant par excellence celui qui actualise ou "réforme" le texte constitutionnel, en en dégageant les riches et extraordinaires possibilités inhérentes à l'indétermination volontaire de la plupart de ses concepts et catégories. Et que la réforme advienne pour faire progresser l'affirmation graduelle des objectifs fondamentaux, et pas pour la faire rétrograder.

Je le réitère: les mises à jour effectuées par l’interprète de sa mission téléologique sont celles qui doivent être envisagées comme prioritaires dans notre modèle de constitutionalisme topique-systématique, surtout lorsque nous reconnaissons que le juge est l'élément culminant des processus de positivation juridique. Dès lors, nous avons besoin de faire confiance et d'octroyer notamment à l'interprète le rôle de promoteur des changements ponctuels de notre Constitution. L'effet hautement bénéfique de cette posture constituera en un gain sensible de prévisibilité juridique, en plus d'une garantie de continuité dans un monde marqué par l'empreinte du changement, signe inéquivoque et irréversible de notre époque changeante (Souvent, trop changeante, comme vu lors de la dramatique crise financière éclose en 2008, par défaut de la réglémentation de l'Etat et du marché).

Parallèlement, force est d'observer que l'idée de révision de la Constitution mérite une compréhension tout à fait restrictive, dans le cas brésilien, de sorte que dans notre système nous ne devons pas passer par une autre révision au sens strict. Autrement dit, celle qui aurait pu être faite l'a déjà été. Désormais, il y a tout simplement l'opportunité de réformes à travers l'exercice du pouvoir d'amender, à côté des "mutations constitutionnelles" réalisées (parcimonieusement) par la voie interprétative, toutes les deux ayant le devoir de ne pas violer lesdits principes sensibles, parmi lesquels les droits et garanties individuels (art. 60 de la CF) et les objectifs fondamentaus de la République Fédérative (art. 3 de la CF). Une telle

${ }^{42}$ in Systemdenken und Systembegriff in der Jurisprudenz, ob. cit. 
prescription résulte du précepte qui veut que, en hiérarchisant, nous devons attribuer à la fois une ouverture et une consistance à l'ordre constitutionnel, qui n'est pas du tout compatible avec le manque de cérémonie avec lequel on pense aux Amendements Constitutionnels. ${ }^{43}$ En effet, il n'existe pas de pays, pour rester dans la métaphore, qui réussisse à travailler en permanence à cœur ouvert. Le Brésil supplante laborieusement le martyre d'un faux réformisme en profusion, en suivant la tendance hautement reprochable de la banalisation du processus d'amendement. On constate sous maints aspects une hyper-inflation de propositions irréfléchies d'amendements à la Constitution, coexistant avec quelques mesures réellement inajournables, dans le cas de réglémentations pas encore faites. Il est cependant permis d'espérer que cet élan démesuré trouve sa fin chez l'interprète dans son exercice majeur de mise à jour et de préservation de la vie, de l'unité et de la justesse de la Constitution et de ses objectifs fondamentaux. Il est essentiel, peut-être plus qu'à n'importe quel autre moment de notre histoire, de promouvoir la fonction psychologique même de stabilisation de la Constitution, en intensifiant le soin d'attribuer une stabilité au système juridique, sur son plan le plus élevé. Tout cela pour produire une révérence coutumière à la Constitution, dans ce que l'on pourrait considérer comme son centre tourné vers l'affirmation de la dignité humaine ${ }^{44}$ (CF, art. 3, III) et, donc, de la transformation vers la justice matérielle. ${ }^{45}$

Le précepte illustratif suivant indique, sans mépriser l'intention du Constituant originaire ou dérivé, qu'il importe de veiller à la souveraineté de la vitalité du système constitutionnel dans sa totalité, en adoptant quand il le faut et avec une extrême parcimonie, la technique de l'exégèse corrective, sans basculer dans la pratique maladroite de l'amputation précipitée. Plus que la "voluntas legislatoris" ou même la "voluntas legis", il faut souligner et rendre organique la volonté de la Constitution (pour évoquer l'expression chère à Hesse). La connexion intime de ce précepte avec les précédents est évidemment indéniable. L’intention du législateur constituant historique apparaît importante mais secondaire. Dans ce contexte, il n'existe pas de norme constitutionnelle originaire inconstitutionnelle (pour des raisons logiques); toutefois, même en face du texte originaire, il est indispensable d'avoir le courage de le corriger afin de préserver la volonté du système dans son esprit supérieur ${ }^{46}$. Ainsi, lorsque dans l'article 243 la Constitution dit par exemple “expropriadas”, elle veut dire « confisquées », dans une compréhension modérément corrective. Il n’est pas difficile de soutenir cette compréhension si nous lisons le dispositif dans sa totalité et surtout si nous le conjuguons avec les autres, en particulier avec l'article 5, XXIV. Plus que l'intention de la norme, c’est la vitalité du système constitutionnel qui est

43 Lors de ses 20 ans, la Constitution possédait 142 dispositifs passibles de réglémentation et, en même temps, plus de 1500 projets d 'Amendements Constitutionnels, et 62 Amendements déjà réalisés. Les chiffres sont éloquents et parlent par eux-mêmes.

${ }^{44}$ Voir Ingo Sarlet in Dignidade da Pessoa Humana e Direitos Fundamentais na Constituição Federal de 1988. 6a ed., Porto Alegre: Livraria do Advogado, 2008.

${ }^{45}$ Voir Dominique Rousseau in Les grandes avancées de la jurisprudence constitutionnelle, in Mélanges J. Robert, L.G.D.J., 1998, p. 297.

${ }^{46}$ Cf. les leçons de Edward C. Black in Handbook on the Construction and Interpretation of the Laws, St. Paul, West Publishing Co., 1896, pp. 48 et 104. 
concernée; ce système représente le produit de l'interaction ou de la convergence irremplaçable entre le texte et l'interprète (cognitivement et volitivement considéré), car nous avons affaire à l'interaction typiquement dialectique. Encore une fois, il convient de souligner que les syllogismes juridiques ne divergent pas, ni aujourd'hui ni depuis toujours, de ceux qu'Aristote nommait dialectiques ${ }^{47}$.

Cependant, on ne doit pas oublier totalement l'intention historique du Constituant, malgré une volonté collective et confuse ${ }^{48}$, dont l'investigation exacte est presque impossible. Il suffit d'évoquer les exemples explicatifs de débats herméneutiques qui se sont produits au Brésil au sujet de la particule "et”" (Serait-elle disjonctive ? Serait-elle additive ?) et la fonction étrange d'un point-virgule, impliquant toujours la réforme de la Sécurité Sociale. Dans les deux hypothèses, la consultation des travaux préparatoires fut déterminante comme instrument d'argumentation. Ainsi, il est des cas où les supposées intentions originelles, modérément ${ }^{49}$ prises en considération, (surtout si elles se montrent massives et résultantes de consensus pas trop nébuleuses) ne peuvent pas tout simplement être méprisées. La plupart du temps, cependant, l'ambiguïté ou l'indétermination exacerbée sont intentionnelles, voir même objets de négociation du processus législatif, de sorte que la quête de la volonté historique s'avère en elle-même franchement insatisfaisante. En plus de cela, nous devons garder à l'esprit les critiques opportunes formulées par Dworkin sur "l'originalisme" (particulièrement lors de l'éducative polémique avec Bork). ${ }^{50}$ La posture littéraliste ou originaliste extrême conduit à l'interprétativisme strict et, à la limite, succombe à l'illusion napoléonienne d'un magistrat serf de la loi et - ce qui est plus grave - nonobéissant au Droit, et particulièrement, à ses objectifs fondamentaux.

Le huitième précepte d’intérprétation constitutionnelle suggéré montre quant à lui que les droits fondamentaux ne doivent pas être appréhendés séparément ou localement comme s'ils étaient tous insérés dans l'article 5 de la Constitution Fédérale. Force est de le voir disséminé dans l'ordonnancement, demandant une vitalisation accentuée, notamment par rapport aux droits de seconde, troisième dimensions, avec involution péremptoirement interdits, même si déguisée en progrès de façon attirante. En effet, une fois reconnu l'un de ces droits fondamentaux par le législateur, sa suppression se montrera "prima facie" inconstitutionnelle. Plus encore: grâce à la clause apportée par la norme générale inclusive authentique, prévue dans le $\S 2$ de l'article 5 (“les droits et les garanties exprimés dans cette Constitution n’en excluent pas d'autres découlant du régime et des principes adoptés par elle...”), sont intégrés de manière implicite à notre répertoire de droits fondamentaux tous ceux consacrés par la meilleure tradition de proclamations des droits humains, des plus anciennes aux plus contemporaines.

\footnotetext{
${ }^{47}$ Topica et Sophistici Elenchi. Oxford: Oxford Classical Texts, 1989.

${ }^{48}$ Voir des impressionantes révélations de Jarbas Passarinho à propos du changement de dernier moment de la redaction de l'article 42 de la CF in Caderno Especial « 20 Anos de Constituição ». O Estado de São Paulo, le 2 octobre 2008.

${ }^{49}$ A propos d'originalisme modéré, voir Laurence Tribe in American Constitutional Law. 3a ed. New York: Foundation Press, 2000, pp. 30-84.

${ }^{50}$ Voir Dworkin v. Bork. Madrid: Civitas, 1989.
} 
Dans cet ordre d'idées, tout applicateur a besoin d'assumer, surtout lorsqu'il a affaire aux droits fondamentaux, la prémisse que les garanties doivent servir de rempart énergique contre le non-accomplissement de précepte fondamental résultant du Pouvoir Public ${ }^{51}$, raison pour laquelle il faut éviter tout résultat interprétatif qui réduise ou affaiblisse, sans motif valable, l'efficacité maximale de droits fondamentaux ${ }^{52}$. Dans ce contexte, il faut que l'exégèse promeuve et concrétise les objectis fondamentaux de notre ordre (article 3ème), susceptibles de fonctionner comme vecteur principal de la compréhension supérieure de toutes les branches du Droit. Plus que in dubio pro libertate, principe précieux dans les rapports du citoyen face au Pouvoir Public, le commandement humanisant selon lequel au profit de la dignité et des objectifs de l'article 3ème de la Constitution il ne peut pas y avoir de doute.

Un autre vecteur utile (dans le meilleur sens du mot) de l'interprétation constitutionnelle est celui qui veut que les principes fondamentaux constitutionnels ne doivent jamais être éliminés mutuellement même s'ils sont en collision à la différence de ce qui se produit, seulement en cas ultime, avec les règles juridiques d'inconstitutionnalité déclarable. Par rapport à celles-ci, lorsque l'antinomie, partielle ou totale, s'impose, une règle supprime l'autre; même si, ici encore, une logique du “tout ou rien” ne s'applique pas, au contraire de ce qui est défendu par d'éminents auteurs (sujet qui ne peut pas être développé dans cette étude). Il faut conserver intact l'esprit des principes et des droits fondamentaux, car ils fonctionnent comme des directives suprêmes révélant une fondamentalité qui se pose comme condition insupprimable de préservation de l'édifice constitutionnel. À titre d'exemple, le principe de la légalité ne sort pas affaibli de l'autonomie juridique attribuée au principe de la moralité, mais au contraire fortifié et élargi ${ }^{53}$. Tout se passe comme si un principe, en relativisant, enrichissait et rendait plus dense le signifié de l'autre. Il s'agit à la fois d'un mouvement de contention et d'expansion.

Il convient de répéter: les principes ne doivent jamais être affaiblis, au risque de mener à l'extinction du système. Pontes de Miranda l'a signalé: "Les règles juridiques formeront système. (...). Cette exigence de systématicité du Droit répond au besoin de cohérence et de consistance dans la conduite humaine, principalement en ce qui concerne la vie en relation" ${ }^{\text {". }}$. Il faut ajouter cependant que les règles ne peuvent pas à elles seules bâtir quelque système que ce soit. Ce sont les principes fondamentaux qui jouent le rôle organique et protéique de structurateurs et d'incitateurs du système, de sorte qu'ils doivent invariablement être interprétés, afin d'être préservés, même s’il est impérieux de les nuancer parce qu'ils ne doivent pas être confondus avec les normes strictes ou les règles, celles-ci - seules celles-ci déclarées inconstitutionnelles, souvent prononcées nulles dans tous les effets, même si non nécessairement, comme déjà vu.

${ }^{51}$ Voir la Loi 9882 de 1999, en particulier les articles 4, 5, 10 et 11;

${ }^{52}$ Sur ce sujet, voir Ingo Sarlet in A Eficácia dos Direitos Fundamentais. 9. ed, Porto Alegre: Livraria do Advogado, 2008.

${ }^{53}$ Sur le lien entre Droit et Moral, voir Robert Alexy, Begriff und Geltung des Rechts, Munich, Verlag Karl Alber, Freiburg, 1992, pp. 15-17.

${ }^{54}$ Comentários à Constituição de 1967 com a Emenda $n^{\circ} 1$ de 1969, Rio de Janeiro: Forense, 1987, p. 39. 
Je dois attirer l'attention à la garde substantielle des principes, plus appropriée aux clameurs de la dignité conjuguée aux autres objectifs fondamentaux et sans que ceux-ci sacrifient celle-là, c'est-à-dire sans succomber au formalisme ou argumentatif ou conceptualisme, dans le sens d'appel seulement à la norme ou à l'ensemble de normes. ${ }^{55}$

Enfin, il convient de souligner que le répertoire présenté n’est pas exhaustif. On pourrait parler, par exemple, du précepte de la prévention, en d'autres mots, le juge doit, tant que possible, agir de façon préventive et en temps d'éviter que l'inconstitutionnalité s'enracine, pour éviter la gêne provoquée par des situations consolidée, malgré le vice grave d'origine. Toutefois, je trouve impérieux de faire allusion, même brièvement, à un dixième devoir herméneutique, à titre de suggestion de catalogue ouvert (il ne s’agit pas de "cânones » évidemment): celui de réaliser, dans les limites du système, une interprétation conforme au «bien de tous » (CF, article 3 , IV) ayant le but d 'une « société libre, juste et solidaire) (CF, article 3ème, I), c'est-à-dire, non-particulariste et, si nécéssaire, pour le faire, même les décisions contraires aux majorités occasionnelles se justifient. Il me semble que l'interprétation du système constitutionnel trouve à ce point un de ses sommets. Ici l'on veut dire que l'intérprétation doit viser à vraiment promouvoir le dévéloppement, la liberté, la justice et la solidarité comme condition pour une fondamentation juridique authentique. À ce précepte interprétatif se trouve indissolublement liée la présomption que le système juridique existe pour l'être humain, et pas le contraire. Toutes les présomptions juridiques sont moins importantes que celle-ci, lors du choix entre les potentiellement très larges alternatives offertes par la Constitution. En résumé, selon ce critère, l'exégète topique-systématique doit déclarer l'inconstitutionnalité matérielle ou substantielle ${ }^{56}$ de toute loi ou acte normatif manifestement hostile ou en litige avec l'ensemble d'objectifs de l'article 3ème de la Constitution brésilienne, sans que, pour autant, il doive se servir des motivations extra-juridiques. Il ne s'agit pas d'adhérer - bien au contraire - à trop de conservatisme textualiste, car si l'inconstitutionnalité se trouve nettement configurée, il n'y a aucun profit à retarder la suppression de la norme viciée, encore que très exceptionnellement les effets de cette déclaration d'inconstitutionnalité puissent être restreints, ou de décider qu'elle ne soit efficace qu'à partir du jugement définitif ou d'un autre moment. Autrement dit, ce devoir exégétique demande une harmonisation continuelle avec la téléologie supérieur de la Constitution, notamment face aux exigences du principe de dignité qui se croise avec la défense du «bien de tous».

Certes, les préceptes interprétatifs, sommairement proposés, ont été référés dans un esprit incitatif, ouvert et coopératif. Je crois qu'on ne doit pas renoncer à l'idéalisme réaliste. Cela impliquerait rénoncer à l'humanité. L’idée que le «bien de tous » soit possible est une belle source d'évolution, même si le scepticisme conformiste ou opportuniste essaye d'affirmer le contraire. Par conséquent, quand nous intériorisons et prenons conscience de tels préceptes, nous serons aptes à adapter la Constitution aux exigences présentes et futures concernant la vraie promotion du dévéloppement humain.

\footnotetext{
55 Voir Riccardo Guatini in Das Fontes às Normas. São Paulo: Quartier Latin, 2005, p. 159.

${ }^{56}$ Voir Juarez Freitas in A Substancial Inconstitucionalidade da Lei Injusta. Rio: Vozes, 1989.
} 


\section{CONCLUSIONS}

(a) Dans le système juridique brésilien, tout juge est, d'une certaine façon, un juge constitutionnel, et il est impossible de ne pas préserver, au maximum, la coexistence pacifique et harmonieuse entre les contrôles diffus et concentrés de constitutionnalité, prenant en considération la concrétisation croissante des objectifs fondamentaux prévus dans l'article 3 de la Constitution Fédérale et ainsi, construire une société libre, juste et solidaire, garantir le dévéloppement; éliminer la pauvrété et la marginalisation et réduire les inégalités sociales et régionales et promouvoir le bien de tous, sans préjugés d'origine, race, sexe, couleur, âge ou d'autre formes de discrimination.

(b) En autre, l'interprétation constitutionnelle est un processus topiquesystématique, de sorte qu'il est impératif, lors de l'examen des cas, d'atteindre une solution d'équilibre dialétique entre le formalisme et la téléologie concrète, en évitant des solutions unilatérales et rigides, adaptant plastiquement la Constitution à ses propres buts supérieurs.

(c) En hiérarchisant prudemment les principes et les règles constitutionnelles, la téléologie doit occuper une place plus importante tout en les situant à la base et au sommet du système, dû à son lien évident avec la primauté des objectifs fondamentaux.

(d) L'interprète constitutionnel se doit d'être le gardien, par excellence, d'une vue proportionnelle des éléments constitutifs de la Charte Constitutionnelle, mais la proportionnalité ne doit pás être entendue seulement comme une adéquation entre les moyens et les fins. Il s'agit de l'interdiction de l'arbitraire par excès et par omission. Proportionnalité signifie, surtout, que nous sommes obligés à sacrifier le minimum pour préserver le maximum de droits.

(e) L'interprète constitutionnel doit considérer largement l'inaliénable pouvoirdevoir d'offrir la tutelle juridictionelle de manière à faciliter, au maximum, l'accès légitime du citoyen, y inclus à la jurisdiction constitutionnelle. Autrement dit, il s'agit de dégager les effets les plus profonds de l'adoption, chez nous, de l'intangible système de la juridiction unique.

(f) L'interprète constitutionnel doit servir à assumer le discours normatif de la Constitution, dans ce qu'elle possède d'éthiquement supérieur, en lui conférant de la sorte à la fois la cohérence interne et l'efficacité sociale, en particulier concernant les objectifs cités dans l'article 3ème de la CF.

(g) L'interprète constitutionnel doit chercher une fondamentation rationnelle et objective pour ses décisions en synchronie avec le système, sans adopter des solutions “contra legem”, bien qu'il exerce une activité consciemment et carrément normative, tout en reconaissant que la technique de la pensée topique ne se distingue pas essentielment de la technique systématique, toutes les deux des facettes du même pouvoir de hiérarchiser et donner de l'anima au système, parmi les plusiers possibilités de sens. 